\title{
Analysis of the clinical value of fractional flow reserve for prognosis evaluation of patients of percutaneous coronary intervention
}

\author{
QINGXIA ZHAO, ZHENG JI, XIA LI, YALI DI, HAOJUN AN, BIN WEI, LIMING YANG and WENSHENG CHEN \\ Department of Cardiology, Tangshan Workers' Hospital, Tangshan, Hebei 063000, P.R. China
}

Received February 27, 2017; Accepted May 22, 2017

DOI: $10.3892 / e t m .2017 .5433$

\begin{abstract}
We investigated the clinical value of fractional flow reserve (FFR) for the prognostic evaluation of patients with percutaneous coronary intervention. We enrolled 120 patients who were admitted to the hospital to undergo percutaneous coronary intervention for acute coronary syndromes between May 2014 and June 2015. The 120 patients were divided into two groups, the observation group and the control group, according to the post-surgery level of FFR. Each cohort contained 60 patients. These patients were divided into the occurrence group $(n=45)$ and the non-occurrence group $(n=75)$, classified according to the occurrence of major adverse cardiovascular events (MACE). There were no statistically significant differences in the comparison of the occurrence rate of MACE within 30 days after surgery, such as lethal or non-lethal myocardial infarction or non-lethal cerebrovascular events, between the observational group and the control group $(\mathrm{P}>0.05)$. For the observation group, the 1-year survival cases were 56 with a survival rate of $93.3 \%$, and 2-year survival cases were 50 with a survival rate of $83.3 \%$. In the control group, the 1-year and 2-year survival cases were respectively 55 and 49 (survival rate of 91.7 and $81.7 \%$ ). The occurrence rates of hyperlipidemia and ratio of patients with a history of smoking and drinking in the occurrence group were significantly higher than those in the non-occurrence group $(\mathrm{P}<0.05)$. The mean arterial pressure in the occurrence group was significantly higher than that in the non-occurrence group $(\mathrm{P}<0.05)$. Heart rate in the occurrence group is significantly faster than that in the non-occurrence group $(\mathrm{P}<0.05)$ and the stenosis degree in the occurrence group was significantly higher than that in the non-occurrence group $(\mathrm{P}<0.05)$. The left ventricular ejection fraction (LVEF) before surgery in the occurrence group was significantly lower than that in the non-occurrence group $(\mathrm{P}<0.05)$. There were
\end{abstract}

Correspondence to: Dr Zheng Ji, Department of Cardiology, Tangshan Workers' Hospital, 27 Cultural Road, Tangshan, Hebei 063000, P.R. China

E-mail: jizheng201608@163.com

Key words: fractional flow reserve, coronary artery, intervention, stenting, prognosis evaluation no remarkable differences in comparison of the pre-treatment FFR between the occurrence group and the non-occurrence group ( $\mathrm{P}>0.05$ ), but the post-treatment FFR in the occurrence group was significantly lower than that in the non-occurrence group $(\mathrm{P}<0.05)$. Increased blood fat, a history of smoking and drinking, augmented mean arterial pressure, accelerated heart rate, severe coronary artery stenosis and the remarkably decreased LVEF were all identified as independent risk factors leading to major adverse myocardial events. The sum of specificity and sensitivity of treatment reached the peak when the post-surgery FFR was 0.875 , the calculated sensitivity was $82.4 \%$, and the specificity was $50.8 \%$. In conclusion, measurement of FFR after percutaneous coronary intervention could not only effectively evaluate the target vessel revascularization, but also predict the occurrence of major adverse myocardial events 1 year after surgery, which could serve as the guidance for clinical treatment.

\section{Introduction}

Coronary atherosclerotic heart disease is a type of severe disease which, due to the organic lumen stenosis or even blockages of coronary arteries, often leads to a decrease in the blood supply of coronary arteries, which can barely satisfy the need of oxygen supply of the myocardium, further resulting in ischemic injury of myocardium (1). The occurrence rate of coronary heart disease is increasing with the social and economic development in China, as well as an improvement in people living conditions. Currently, percutaneous coronary intervention is the most effective method for the treatment of acute coronary syndromes. Previous research has confirmed the insufficient effect of coronary arteriography in identifying whether the coronary artery stenosis patients are concomitant with the myocardial ischemia, especially for patients with moderate coronary artery stenosis (2). Severe myocardial ischemia is believed to be a key factor leading to the death of patients and poor prognosis (3).

Fractional flow reserve (FFR) is the ratio of maximum blood volume that can be achieved in the lesion vessels to the normal reference value in the presence of coronary artery stenosis (4). In the presence of significant coronary artery stenosis, some of the kinetic energy of the blood flow through the stenosis parts becomes converted into thermal energy with a decrease in the pressure of blood flow, thus leading to 
a great loss of energy. Therefore, we can indirectly estimate the influence of stenosis on the myocardial perfusion by measuring the changes in pressure (5). Since the FFR is free from the influence of systemic hemodynamics and its clinical normal reference value is already established, the FFR can be applied to better guide the percutaneous coronary intervention to achieve better clinical efficacy (6). In the present study, the aim was to investigate the predictive value of FFR for the prognosis of patients who received the percutaneous coronary intervention due to acute coronary syndromes. Specific procedures are reported below.

\section{Materials and methods}

Sample selection. We enrolled 120 patients who were admitted to the hospital to receive coronary stenting for acute coronary syndromes between May 2014 and June 2015. Before enrollment, 120 patients were asked about their conditions in detail, including the past medical history, age, height, weight, the history of smoking and drinking, combined hypertension, diabetes, or chronic obstructive pulmonary diseases, cardiac functions, previous physical condition. The measurement of vital signs such as blood pressure, heart rate, breathing rates were performed, the combination examination of electrocardiogram and biochemical test for determining the indexes of myocardial enzyme, troponin, B-type natriuretic peptide, blood routine examination and blood clotting function was also conducted. Fasting elbow venous blood was drawn for assaying blood glucose, blood fat and hepatic as well as renal functions. All the patients could afford the cost for the treatment of cardiac intervention by implanting the stent and the cost for assaying the FFR, which was performed before and after intervention therapy. Before enrollment, all patients had signed the written informed consent of intervention therapy and the written informed consent of enrollment, and the present study was approved by the Ethics Committee of the Tangshan Hospital.

The exclusion criteria were: i) patients who had history of open coronary artery bypass surgery, ii) patients who were in cardiac shock, iii) patients who were diagnosed with myocardial diseases, iv) patients with ventricular hypertrophy, v) patients with severe renal and hepatic dysfunction and vi) patients who were allergic to the drugs used in the present study. The enrolled patients were divided into two groups, the observation group and the control group, according to the post-surgery levels of FFR. Each group contained 60 patients and general characteristics of both groups are shown in Table I, in which there was no statistically significant difference in the comparison of sex, age, ratio of hyperlipidemia, ratio of patients with a history of smoking and drinking, height, weight, mean arterial pressure, heart rate, stenosis degree of coronary artery, pre-surgery left ventricular ejection fraction (LVEF) and pre-surgery FFR between the two groups $(\mathrm{P}>0.05)$. Additionally, these patients were divided into two groups, the occurrence group $(n=45)$ and the non-occurrence group ( $n=75)$, according to the occurrence of major adverse cardiovascular events (MACE).

Treatments. For all enrolled patients, we performed intensive care, which closely monitored their conditions by a 24-h ECG, ensured bed rest, discharges of urinary and fecal matter in bed and a light diet. In addition, we administered sedation to dull the pain and nitroglycerine to dilate their vessels, aspirin for anti-platelet therapy, low-molecular-weight heparins by subcutaneous injection for anti-coagulation therapy and a combination of angiotensin and $\beta$-receptor blocker for regulating the pressure. These patients were divided into two groups according to the pre-surgery levels of FFR, i.e., the control group (FFR $>0.80)$ and the observation group (FFR $\leq 0.80)$. For the control group, conservative treatment by a single administration of drugs was applied, while for the observation group, stenting for revascularization was performed in addition to the relevant conservative treatment.

Observation indexes. All patients were followed up for 2 years either by telephone or through outpatient service. During the follow-up period, we compared the occurrence rates of MACE in 30 days after surgery and the Kaplan-Meier survival analysis curves between the observation group and the control group, as well as the general materials and FFR before and after surgery between the occurrence group and the non-occurrence group. We also performed multi-factor logistic regression analysis for the occurrence of MACE 30 days after surgery, and analyzed the predictive value of post-surgery FFR for the occurrence of MACE in 1 year.

Assessment methods. i) Two dimensional ultrasonography was applied in the ECG to assay the relevant data of the enrolled patients' heart, including the left arterial diameter and LEVF, and used the pulsed wave Doppler to investigate the blood flow and its orientation in the bicuspid valve, the tricuspid valve, aortic valve and the pulmonary valve; ii) coronary angiography, coronary angiography was performed using the standard Judkins method for all the enrolled patients, i.e., patients that had signed the written informed consent before surgery, and the physicians selected the radial artery in order to perform the puncture when patients were under local anesthesia. Thereafter, according to the results of coronary angiography, patients whose one-vessel stenosis degree was over $50 \%$ would be diagnosed as coronary heart disease; while the coronary artery TIMI blood flow refers to the condition of blood flow in the infarction-related sites during the onset of acute myocardial infarction, which was divided into five degrees ( 0 to IV), and a lower degree represented more severe infarction and iii) measuring the FFR: this process was performed using the pressure sensor, in which the FFR value was set to 1.00 when the changes of aortic pressure were paralleled with the pressure curve of the pressure guidewire. Based on the settings, we investigated the variation of FFRs before and after treatment and the minimum and mean of the FFRs, in whom the reference value of FFR was set as 0.80 . For patients with the FFR higher than 0.80 , a conservative therapy by administration of drugs could be simply applied. The FFR assay and the coronary angiography were performed strictly under the instructions of qualified clinical physicians at this hospital with the work experience longer than 10 years.

Statistical analysis. SPSS 13.0 software (SPSS, Inc., Chicago, IL, USA) was applied to the statistical process. Nonparametric test was performed for the non-normally distributed data. For 
Table I. Comparison between the observation group and the control group.

\begin{tabular}{|c|c|c|c|c|}
\hline Items & Observation group & Control group & t-test or $\chi^{2}$ & P-value \\
\hline Sex (male/female) & $36 / 24$ & $35 / 25$ & 0.034 & 0.853 \\
\hline Age (years) & $62.1 \pm 1.8$ & $62.3 \pm 1.8$ & 0.609 & 0.544 \\
\hline Ratio of hyperlipidemia (\%) & $40(66.7 \%)$ & $41(68.3 \%)$ & 0.038 & 0.845 \\
\hline Ratio of patients with a history of smoking and drinking (\%) & $26(43.3 \%)$ & $27(45.0 \%)$ & 0.034 & 0.854 \\
\hline Height $(\mathrm{cm})$ & $163.2 \pm 2.1$ & $163.3 \pm 2.1$ & 0.261 & 0.795 \\
\hline Weight (kg) & $68.5 \pm 1.4$ & $68.6 \pm 1.5$ & 0.378 & 0.706 \\
\hline Mean arterial pressure $(\mathrm{mmHg})$ & $125.6 \pm 2.5$ & $126.1 \pm 2.6$ & 1.074 & 0.285 \\
\hline Heart rate (beats/min) & $98.5 \pm 2.3$ & $98.6 \pm 2.4$ & 0.233 & 0.816 \\
\hline Stenosis degree of coronary artery $(\%)$ & $65.3 \pm 2.9$ & $65.4 \pm 3.0$ & 0.186 & 0.853 \\
\hline Pre-surgery LVEF (\%) & $43.2 \pm 1.8$ & $43.3 \pm 1.8$ & 0.304 & 0.761 \\
\hline Pre-surgery FFR (\%) & $0.72 \pm 0.05$ & $0.73 \pm 0.06$ & 0.992 & 0.323 \\
\hline
\end{tabular}

LVEF, left ventricular ejection fraction; FFR, fractional flow reserve.

normally distributed data, a t-test was performed for intergroup comparison of the mean. Measurement data were presented as mean \pm standard variation, and the ROC curve was drawn for assessing the predictive value of FFR after surgery for the occurrence of MACE in 1 year. The $\chi^{2}$ test was applied to the intergroup comparison of rates, and the log-rank test was performed for statistics of intergroup data and the survival curve was accordingly established. A multi-factor logistic regression analysis was carried out for analyzing the relevant influential factors. $\mathrm{P}<0.05$ suggested that the difference was statistically significant.

\section{Results}

Comparison of MACE occurrence in 30 days post-surgery between the observation group and the control group. For the MACE occurrence in 30 days post-surgery of the observation group, there was 1 death (1.7\%), 11 cases with non-lethal myocardial infarction (18.3\%) and 13 cases with non-lethal cerebrovascular event $(21.7 \%)$. While for that of the control group, there were 3 deaths $(5.0 \%), 12$ cases with non-lethal myocardial infarction $(20.0 \%)$ and 15 cases with non-lethal cerebrovascular event (25.0\%) (Fig. 1). There were no statistically significant differences in the comparison of the MACE occurrence between the groups.

Analysis of the Kaplan-Meier survival curves of the observation group and the control group. For the observation group, the 1-year survival cases were 56 with a survival rate of $93.3 \%$, and 2-year survival cases were 50 with a survival rate of $83.3 \%$. For the control group, the 1-year and 2-year survival cases were, respectively, 55 and 49 (the survival rates were 91.7 and $81.7 \%$ ) (Fig. 2).

General comparison between the occurrence group and the non-occurrence group. There was no statistically significant difference in comparison of sex, age, height and weight between the occurrence group and the non-occurrence group $(\mathrm{P}>0.05)$. Both of the occurrence rate of hyperlipidemia and

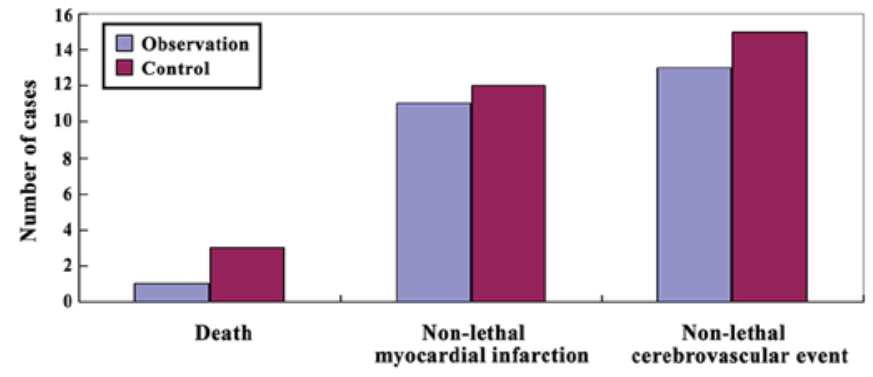

Figure 1. Comparison of the MACE occurrence in 30 days after surgery between the observation group and the control group. MACE, major adverse cardiovascular events.

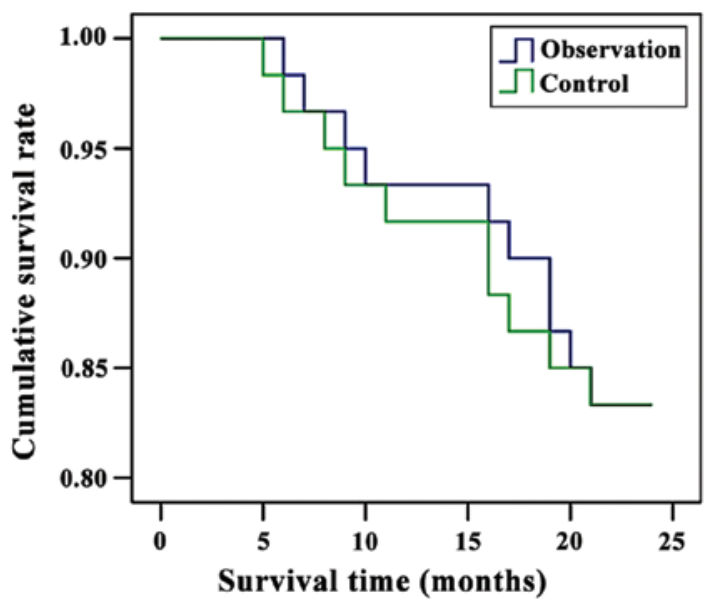

Figure 2. Analysis of the Kaplan-Meier survival curves of the observation group and the control group.

ratio of patients with a history of smoking and drinking in the occurrence group were significantly higher than those in the non-occurrence group $(\mathrm{P}<0.05)$; the mean arterial pressure in the occurrence group was significantly higher than that in the non-occurrence group $(\mathrm{P}<0.05)$; the heart rate in the occurrence group is significantly faster than that in the non-occurrence 
Table II. Results between the occurrence group and the non-occurrence group.

\begin{tabular}{|c|c|c|c|c|}
\hline Items & Occurrence group & Non-occurrence group & t-test or $\chi^{2}$ & P-value \\
\hline Sex (male/female) & $25 / 20$ & $40 / 35$ & 0.056 & 0.813 \\
\hline Age (years) & $62.6 \pm 1.9$ & $62.5 \pm 1.9$ & 0.279 & 0.781 \\
\hline Ratio of hyperlipidemia (\%) & $41(91.1 \%)$ & $51(68.0 \%)$ & 7.155 & 0.007 \\
\hline Ratio of patients with a history of smoking and drinking $(\%)$ & $40(88.9 \%)$ & $50(66.7 \%)$ & 7.407 & 0.006 \\
\hline Height $(\mathrm{cm})$ & $163.6 \pm 2.1$ & $163.6 \pm 2.0$ & 0.000 & 1.000 \\
\hline Weight (kg) & $68.6 \pm 1.4$ & $68.5 \pm 1.5$ & 0.362 & 0.718 \\
\hline Mean arterial pressure $(\mathrm{mmHg})$ & $141.6 \pm 5.1$ & $126.3 \pm 2.5$ & 21.988 & $<0.001$ \\
\hline Heart rate (beats/min) & $108.7 \pm 3.3$ & $93.5 \pm 2.3$ & 29.678 & $<0.001$ \\
\hline Stenosis degree of coronary artery $(\%)$ & $75.8 \pm 2.8$ & $63.2 \pm 3.1$ & 22.336 & $<0.001$ \\
\hline Pre-surgery LVEF (\%) & $41.3 \pm 1.7$ & $46.8 \pm 1.9$ & 15.956 & $<0.001$ \\
\hline
\end{tabular}

LVEF, left ventricular ejection fraction.

Table III. Comparison of FFRs before and after treatment between the occurrence group and the non-occurrence group (mean \pm standard variation).

\begin{tabular}{lcc}
\hline Items & Before treatment & After treatment \\
\hline Occurrence group & $0.75 \pm 0.11$ & $0.83 \pm 0.02$ \\
Non-occurrence group & $0.75 \pm 0.10$ & $0.93 \pm 0.05$ \\
t-test & 0.000 & 12.799 \\
P-value & 1.000 & $<0.001$ \\
\hline
\end{tabular}

FFR, fractional flow reserve.

group $(\mathrm{P}<0.05)$; the stenosis degree in the occurrence group was significantly higher than that in the non-occurrence group $(\mathrm{P}<0.05)$; the LVEF before surgery in the occurrence group was significantly lower than that in the non-occurrence group $(\mathrm{P}<0.05)$ (Table II).

Comparison of FFRs before and after treatment between the occurrence group and the non-occurrence group. There were no statistically significant differences in the comparison of FFRs before the treatment between the occurrence group and the non-occurrence group $(\mathrm{P}>0.05)$. After treatment, the FFR of the the occurrence group was significantly lower than that of the non-occurrence group $(\mathrm{P}<0.05)$ (Table III).

Multi-factor logistic regression analysis for the occurrence of MACEs in 30 days after surgery. The multi-factor logistic regression analysis was performed with the MACE in 30 days post-surgery as the dependent variable, which indicates that the increased blood fat, a history of smoking and drinking, augmented mean arterial pressure, accelerated heart rate, severe coronary artery stenosis and the remarkably decreased LVEF were all identified as independent risk factors leading to MACE (Table IV).

Analysis of predict value of the post-surgery FFR for the MACE occurrence in 1 year. In the present study, we established the ROC curve with the post-surgery FFR as the measurement vector, the occurrence of MACE as the state variable and the reference for grouping and 1 as the defined value. The AUC of ROC was 0.716 , in which P-value was 0.005 , indicating that the sum of specificity and sensitivity of treatment reached its peak when the post-surgery FFR was 0.875 , the calculated sensitivity was $82.4 \%$, and the specificity was $50.8 \%$ (Fig. 3).

\section{Discussion}

Previously, coronary angiography was frequently applied to investigate the stenosis of coronary artery lesion and the efficiency in perioperative assessment of the efficacy for patients with acute coronary syndromes, failing to effectively evaluate the condition of blood flow in the distal parts of the stenosis vessels, causing less accurate understanding of the patient's conditions before surgery (7), or even unnecessary stenting procedures for patients with no severe myocardial ischemic coronary diseases (8). These actions results in a great loss of medical resources, and may even affect the prognosis of patients. FFR, which is mainly applicable to those with stenosis lesion of coronary artery (9), refers to a ratio of the maximum blood volume that can be achieved in the lesion vessels to the maximum value in the same site under the normal physiological conditions (10). FFR is free from the variations of heart rate, blood pressure, and myocardial contractility. In clinical practice, the normal reference value of FFR is set to 1.0 (11). Moreover, FFR is apt to operate and can be repeatedly measured. Thus, it can serve as the functional index for assessing the coronary artery stenosis, which has been widely used by physicians of the cardiology department for decision of intervention therapy (12).

Previous studies have confirmed that FFR of 0.8 , can serve as a clinical reference value for the decision to perform the intervention therapy of stenting. In the present study, we divided the enrolled patients into the observation group (FFR $>0.8)$ and the control group (FFR $\leq 0.8)$ and set 0.8 as the clinical reference value for the decision to perform the stenting procedures. The results showed that there was no statistically significant difference in the comparison of MACE in 30 days 
Table IV. Multi-factor logistic regression analysis for the occurrence of MACE 30 days after surgery.

\begin{tabular}{lcccc}
\hline Items & $\mathrm{B}$ & $\mathrm{s}$ & $\mathrm{OR}$ & P-value \\
\hline Sex (male/female) & 0.358 & 0.628 & 2.336 & 0.219 \\
Age (years) & -421 & 0.602 & 0.658 & 0.459 \\
Ratio of hyperlipidemia (\%) & 1.653 & 0.853 & 0.206 & 0.031 \\
Ratio of patients with a history of smoking and drinking (\%) & 1.596 & 0.758 & 0.196 & 0.030 \\
Height (cm) & 0.325 & 0.599 & 2.321 & 0.218 \\
Weight (kg) & 0.336 & 0.621 & 2.336 & 0.231 \\
Mean arterial pressure (mmHg) & 1.360 & 0.862 & 0.235 & 0.042 \\
Heart rate (beats/min) & 1.625 & 0.758 & 0.125 & 0.006 \\
Stenosis degree of coronary artery (\%) & 1.456 & 0.869 & 0.214 & 0.011 \\
Pre-surgery LVEF (\%) & 1.038 & 0.678 & 0.209 & 0.036
\end{tabular}

MACE, major adverse cardiovascular events; LVEF, left ventricular ejection fraction.

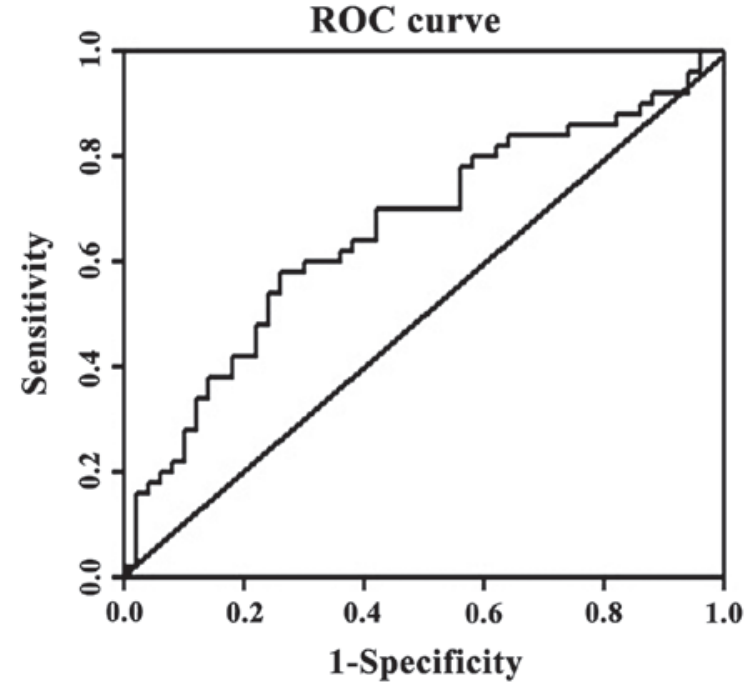

Figure 3. The ROC curve for predicting the occurrence of MACE 1 year post-surgery of FFR. MACE, major adverse cardiovascular events; FFR, fractional flow reserve.

after surgery between the two groups, such as the ratio of deaths, non-lethal myocardial infarction cases and non-lethal cerebrovascular cases. The 1-year and 2-year survival rates in both groups were higher than 90 and $80 \%$, respectively, which was in accordance with the results of studies conducted by Fröhlich et al and Varho et al $(13,14)$. Such results indicate that determining the implementation of coronary artery stenting by the FFR value can achieve better efficacy in guiding clinical practice, which can effectively reduce the ratio of less considered stenting procedures, facilitate the functional revascularization, and minimize the surgical risk and the adverse reaction due to the application of a contrast agent (15). Despite the confirmed positive reference value of FFR in the selection of therapeutic strategies of coronary artery stenting, some patients that received the treatment strictly under the guidance of FFR still suffered the MACE after surgery, such as death, non-lethal myocardial infarction and non-lethal cerebrovascular events. In the present study, we mainly investigated MACE in 30 days after surgery, and found that there were
45 cases with MACE in varying degrees, especially the nonlethal myocardial infarction cases and cases with non-lethal cerebrovascular events (16). Both the occurrence rate of hyperlipidemia and ratio of patients with a history of smoking and drinking in the occurrence group were significantly higher than those in the non-occurrence group. The mean arterial pressure in the occurrence group was significantly higher than that in the non-occurrence group. The heart rate in the occurrence group is significantly faster than that in the non-occurrence group and the stenosis degree in the occurrence group was significantly higher than that in the non-occurrence group. The LVEF before surgery in the occurrence group was significantly lower than that in the non-occurrence group. These results indicate that patients with increased blood fat, a history of smoking and drinking, augmented mean arterial pressure, accelerated heart rate, severe coronary artery stenosis and the remarkably decreased LVEF were more susceptible to MACE in 30 days after surgery, which is coincident with the results of Park et al (17). Through multi-factor logistic regression analysis for the occurrence of MACE in 30 days after surgery, we found that increased blood fat, a history of smoking and drinking, augmented mean arterial pressure, accelerated heart rate, severe coronary artery stenosis and remarkably decreased LVEF were all independent risk factors leading to the MACE in 30 days after surgery (18). It was further confirmed in the present study that patients with the above risk factors, though being treated strictly under the guidance of FFR, would have the significantly increased ratio of MACE in 30 days after surgery.

In addition, the FFR can serve to assess the efficacy immediately after the stenting procedures in clinical practice, especially the target-vessel revascularization after surgery. Previous studies have confirmed that the FFR less than 0.9 (19), which indicates insufficient dilation of stent or poor target-vessel revascularization, but the FFR higher than 0.90 suggests the desired clinical efficacy and better prognosis of patients. In the present study, for investigating the MACE occurrence in 30 days after surgery, we compared the FFRs before treatment and found that there was no statistically significant difference in the comparison of FFR before 
treatment between the occurrence group and the non-occurrence group and that the FFRs were all significantly lower than the normal range. After treatment, the FFR in the occurrence group was lower than that in the non-occurrence group (the average FFR was higher than 0.90 ), which is coincident with the result of Corban et al (20). For further investigation of the predictive value of the post-surgery FFR for the occurrence of MACE 1 year after surgery, we established the ROC curve with the post-surgery FFR as the measurement vector, and the occurrence of MACEs as state variable and the reference for grouping and 1 as the defined value. The AUC of ROC was 0.716 , in which P-value was 0.005 , which indicates that the sum of specificity and sensitivity of treatment reached its peak when the post-surgery FFR was 0.875 , and the calculated sensitivity was $82.4 \%$, and the specificity was $50.8 \%$. Thus, we believe that the MACE in 30 days after surgery would be significantly decreased and increased, respectively, when the post-surgery FFR reached 0.875 , and the prognosis of patients would be significantly improved when the post-surgery FFR surpassed 0.875 .

In conclusion, the measurement of FFR after percutaneous coronary intervention could not only effectively evaluate the target vessel revascularization, but also predict the occurrence of major adverse myocardial events 1 year after surgery, which could serve as guidance for clinical treatment.

\section{Acknowledgements}

The present study was funded by the Hebei Province Planning Commission (no. 20150944).

\section{References}

1. Park SH, Jeon KH, Lee JM, Nam CW, Doh JH, Lee BK, Rha SW, Yoo KD, Jung KT, Cho YS, et al: Long-term clinical outcomes of fractional flow reserve-guided versus routine drug-eluting stent implantation in patients with intermediate coronary stenosis: five-year clinical outcomes of DEFER-DES trial. Circ Cardiovasc Interv 8: e002442, 2015.

2. Maehara A, Ben-Yehuda O, Ali Z, Wijns W, Bezerra HG, Shite J, Généreux P, Nichols M, Jenkins P, Witzenbichler B, et al: Comparison of stent expansion guided by optical coherence tomography versus intravascular ultrasound: the ILUMIEN II study (observational study of optical coherence tomography $[\mathrm{OCT}]$ in patients undergoing fractional flow reserve [FFR] and percutaneous coronary intervention). JACC Cardiovasc Interv 8: 1704-1714, 2015.

3. Vos NS, van der Schaaf RJ, Amoroso G, Herrman JP, Patterson MS, Slagboom T and Vink MA: REVascularization with paclitaxEL-coated balloon angioplasty versus drug-eluting stenting in acute myocardial infarcTION-a randomized controlled trial: rationale and design of the REVELATION trial. Catheter Cardiovasc Interv 87: 1213-1221, 2016.

4. Cho S, Kim JS, Ha J, Shin DH, Kim BK, Ko YG, Choi D, Jang Y and Hong MK: Three-dimensional optical coherence tomographic analysis of eccentric morphology of the jailed side-branch ostium in coronary bifurcation lesions. Can J Cardiol 32: 234-239, 2016.

5. Doh JH, Nam CW, Koo BK, Park SH, Lee JH, Han JK, Yang HM, Lim HS, Yoon MH, Cho YK, et al: Long-term patient-related and lesion-related outcomes after real-world fractional flow reserve use. J Invasive Cardiol 27: 410-415, 2015.
6. De Maria GL, Cuculi F,Patel N, Dawkins S,Fahrni G, Kassimis G, Choudhury RP, Forfar JC, Prendergast BD, Channon KM, et al: How does coronary stent implantation impact on the status of the microcirculation during primary percutaneous coronary intervention in patients with ST-elevation myocardial infarction? Eur Heart J 36: 3165-3177, 2015.

7. Wijns W, Shite J, Jones MR, Lee SW, Price MJ, Fabbiocchi F, Barbato E, Akasaka T, Bezerra H and Holmes D: Optical coherence tomography imaging during percutaneous coronary intervention impacts physician decision-making: ILUMIEN I study. Eur Heart J 36: 3346-3355, 2015.

8. Kimura Y, Tanaka N, Okura H, Yoshida K, Akabane M, Takayama T, Hirayama A, Tada T, Kimura T, Takano H, et al: Characterization of real-world patients with low fractional flow reserve immediately after drug-eluting stents implantation. Cardiovasc Interv Ther 31: 29-37, 2016.

9. Reith S, Battermann S, Hellmich M, Marx N and Burgmaier M: Correlation between OCT-derived intrastent dimensions and fractional flow reserve measurements after coronary stent implantation and impact on clinical outcome. J Invasive Cardiol 27: 222-228, 2015.

10. Courand PY, Dementhon J, Rioufol G and Finet G: Very late neoatherosclerotic plaque rupture in drug-eluting stent restenosis. J Cardiovasc Med (Hagerstown) 16 (Suppl 1): 27-28, 2015.

11. Ito T, Tani T, Fujita $\mathrm{H}$ and Ohte $\mathrm{N}$ : Relationship between fractional flow reserve and residual plaque volume and clinical outcomes after optimal drug-eluting stent implantation: insight from intravascular ultrasound volumetric analysis. Int J Cardiol 176: 399-404, 2014

12. McNeice AH, McAleavey NM and Menown IB: Advances in clinical cardiology. Adv Ther 31: 837-860, 2014.

13. Fröhlich GM, Redwood S, Rakhit R, MacCarthy PA, Lim P, Crake T, White SK, Knight CJ, Kustosz C, Knapp G, et al: Long-term survival in patients undergoing percutaneous interventions with or without intracoronary pressure wire guidance or intracoronary ultrasonographic imaging: a large cohort study. JAMA Intern Med 174: 1360-1366, 2014.

14. Varho V, Karjalainen PP, Ylitalo A, Airaksinen JK, Mikkelsson J, Sia J, Pietilä M and Kiviniemi TO: Transthoracic echocardiography for non-invasive assessment of coronary vasodilator function after DES implantation. Eur Heart J Cardiovasc Imaging 15: 1029-1034, 2014.

15. Dörr R, Stumpf J, Dalibor J, Simonis G and Spitzer SG: Percutaneous coronary intervention versus bypass surgery in patients with diabetes and multivessel coronary disease. Coronary revascularization after FREEDOM. Herz 39: 331-342, 2014 (In German).

16. Onuma Y, Dudek D, Thuesen L, Webster M, Nieman K, Garcia-Garcia HM, Ormiston JA and Serruys PW: Five-year clinical and functional multislice computed tomography angiographic results after coronary implantation of the fully resorbable polymeric everolimus-eluting scaffold in patients with de novo coronary artery disease: the ABSORB cohort a trial. JACC Cardiovasc Interv 6: 999-1009, 2013.

17. Park SJ, Ahn JM, Park GM, Cho YR, Lee JY, Kim WJ, Han S, Kang SJ, Park DW, Lee SW, et al: Trends in the outcomes of percutaneous coronary intervention with the routine incorporation of fractional flow reserve in real practice. Eur Heart J 34: 3353-3361, 2013.

18. Rao VU, Pavlov A, Klearman M, Musselman D, Giles JT, Bathon JM, Sattar N and Lee JS: An evaluation of risk factors for major adverse cardiovascular events during tocilizumab therapy. Arthritis Rheumatol 67: 372-380, 2015.

19. Matsuo A, Fujita H, Tanigaki T, Shimonaga T, Ueoka A, Tsubakimoto Y, Sakatani T, Kimura S, Inoue K and Kitamura M: Clinical implications of coronary pressure measurement after stent implantation. Cardiovasc Interv Ther 28: 170-177, 2013.

20. Corban MT, Eshtehardi P and Samady H: Fractional flow reserve for the assessment of complex multivessel disease in a patient after hybrid coronary revascularization. Catheter Cardiovasc Interv 81: 1169-1173, 2013.

This work is licensed under a Creative Commons Attribution-NonCommercial-NoDerivatives 4.0 International (CC BY-NC-ND 4.0) License. 